\title{
Erratum to: A modified Lowe-Andersen thermostat for a hard sphere fluid
}

\author{
Martijn G. Verbeek ${ }^{\mathrm{a}}$ \\ Hoorn, The Netherlands \\ Original article: Eur. Phys. J. E (2019) 42: 60, https://doi.org/10.1140/epje/i2019-11828-5 \\ Received 16 May 2019 \\ Published online: 28 June 2019 \\ (c) EDP Sciences / Società Italiana di Fisica / Springer-Verlag GmbH Germany, part of Springer Nature, \\ 2019
}

There are two small errors at the beginning of sect. 2 "Theoretical background".

The text: "Particle $i$ has mass $m_{i}$, diameter $\sigma$ and pre-collisional velocity $v_{i}$. Particle $j$ has mass $m_{j}$, diameter $\sigma$ and pre-collisional velocity $v_{j}$. " should read instead as follows: "Particle $i$ has mass $m_{i}$, diameter $\sigma$ and pre-collisional velocity $\vec{v}_{i}$. Particle $j$ has mass $m_{j}$, diameter $\sigma$ and pre-collisional velocity $\vec{v}_{j} . "$

The author apologizes for the inconvenience.

\footnotetext{
${ }^{a}$ e-mail: gustafverbeek@gmail.com
} 